\title{
WHY CASE STUDY RESEARCH? INTRODUCTION TO THE FIELD \\ GUIDE TO CASE STUDY RESEARCH IN TOURISM, HOSPITALITY, AND LEISURE
}

Kenneth F. Hyde, Chris Ryan and Arch G. Woodside

\begin{abstract}
This chapter is a general introduction to the field of case study research in tourism, hospitality, and leisure. The chapter presents a brief review of the literature on the intra-individual logic of case study research. The chapter describes the "four horsemen" for doing case study research: accuracy, generality, complexity/coverage, and value/impact. Examples in the chapter that illustrate this perspective for undertaking case study research may impassion the reader to read through the field guide and personally engage in case study research - at least that is the hope of the editors of this field guide.
\end{abstract}

Keywords: Accuracy; complexity; generality; intra-individual; interindividual; qualitative comparative analysis

Field Guide to Case Study Research in Tourism, Hospitality and Leisure Advances in Culture, Tourism and Hospitality Research, Volume 6, 1-10 Copyright $(\mathcal{C} 2012$ by Emerald Group Publishing Limited All rights of reproduction in any form reserved ISSN: 1871-3173/doi:10.1108/S1871-3173(2012)0000006003 


\section{INTRODUCTION}

If you receive exceptional training and stay active in studying and undertaking behavioral research for a few decades (focusing and practicing for 10,000 hours, see Gladwell, 2008, on this point), you come to realize that you want it all: the ability to describe, explain, and generalize the complexities in the antecedents and nature of the behavior of each individual in your studies. Advances in theory, methods, and technologies in case study research are now transforming this desire into reality.

Rationales for case-based research versus variable-based research should and can go beyond a list of advantages and disadvantages of each general method. Case-based research corrects for substantial - and often fatal flaws in variable-based research if the researcher seeks to understand the behavior of individuals. The prior sentence has profound implications for the study of tourism, hospitality, and leisure.

This introduction offers a brief perspective on the necessity of embracing a case-based research stance for understanding tourism, hospitality and leisure behaviour. Before providing the general rational for the necessity of case-based research for describing and explaining individual behavior, consider the following two observations.

\footnotetext{
Consider the example of a businessman who appeared in one of our studies. He is the chief executive of a Fortune 500 corporation who, when describing the trip that he took to a company location in Florida, reported that he flew first class, he rented a Lincoln [car] on arrival, and stayed at the VIP floor on one of the major upscale hotels. He said, in fact, in an interview, "I worked to get to this position and I deserve it." But later on we talked about a vacation trip that he took to Florida with his wife. They flew People Express, their car was a Rent-A-Wreck, and they stayed at the Days Inn. The gentleman was the same, the psychographics were the same, and he was the same person. These trips were in fact three weeks apart. But they were entirely different occasions, different events, and different decisions. We must, in looking at strategic planning in our business, be more concerned about how the decision is made, not just the person who makes it. (Davidson, 1985, p. 106)
}

Here is the second observation. Several national surveys $(n=3,500$ annually) indicate that the average adult American takes one trip by air annually (mean $=1.00$, standard error of the mean $=.02$ ). Yet, $68 \%$ of adult Americans take no trips by air while $3 \%$ of adult Americans take $30 \%$ of the air trips annually (Woodside, 2011). Interpreting averages frequently leads to misleading conclusions.

The point about averages also applies to interpreting multiple regression analysis (MRA) and structural equation models (SEMs). Any one model 
from MRA or SEM fails to be applicable for most to all of the individuals whose answers were used in constructing and testing the goodness of fit of the model. Several explanations are relevant that support this conclusion.

One explanation: each individual variable that is significant statistically in an MRA model is rarely necessary for achieving a high score on a dependent variable for two reasons. First, due to moderately high correlations among the independent variables in an MRA, an independent variable in an MRA is frequently replaceable by a second independent variable that does not enter into predicting a dependent variable in a stepwise model because of the substantial correlation between the two independent variables.

Second, among the typically three to seven independent variables that are significant in entering a model via MRA or SEM, the particular combination of these three to seven may not apply for any one individual in the sample used to empirically test the model. The significant net effects (absolute sized standardized beta $>.10$ typically) for one or more of the seven variables are inaccurate in predicting the behavior of most to all individuals in the data set used to generate the model.

Consequently, any one "key success factor" to reaching an outcome (e.g., a high score on an independent variable) is not necessary for achieving a high score on a dependent variable. In practice, a correlation of each key success factor with a dependent variable ranges from .20 to .40 ; consequently, the coefficient of determination of the relationships ranges from .04 to .16. A key success factor is not sufficient or even necessary for success. The success factor may hint at one step relevant for some individual to take to reach success (i.e., to achieving a high score on a dependent variable) yet might be not sufficient for achieving success for any one individual.

Rather than thinking of key success factors in achieving a high score for a dependent variable, thinking about building and testing theories to explain "multiple (alternative) key success paths" is both inspirational, practical, and now being done (see Ragin, 2008). Fuzzy-set qualitative comparative analysis (see fsQCA.com) is the principal technique for testing the efficacy of such paths (i.e., "conjunctive statements" or "causal recipes") in modeling individuals, by identifying the paths relevant for each of them.

\section{THE PARADIGM SHIFT TO INTRA-INDIVIDUAL THEORY AND ANALYSIS}

The thought might come to mind that including interaction terms only in regression modeling might be useful for accounting for causal recipe effects 
on a dependent variable. Yes, two- and three-way interactions among independent variables often are significant statistically but such expressions may not be relevant to any one individual or to most individuals in the survey data set. Also, interpreting the meaning of a three-way, four-way, or five-way interaction expression is hard to do - "intractable" comes readily to mind here.

Most research methods (and the dominant logic) in tourism, hospitality, and leisure research employ inter-individual analyses (e.g., empirical positivistic methods building from matrix algebra such as analysis of variance, MRA, and SEM). "However, as shown by classical mathematicalstatistical theorems (the ergodic theorems), such analyses do not provide information for, and cannot be applied at the level of the individual, except in rare occasions when the processes of interest meet certain stringent conditions" (Molenaar \& Campbell, 2009, p. 112). In mathematics, the term "ergodic" describes a dynamical system which, broadly speaking, has the same behavior averaged over time as averaged over space. Molenaar and Campbell (2009, p. 116) conclude, "We are at the brink of a major reorientation in psychological methodology, in which the focus is on the variation characterizing time-dependent psychological processes occurring in the individual human subject .... Legitimate generalization to the wider population is then achieved through identification of subsets of similar individuals. Given the finding that inter-individual variation often cannot be equated to intra-individual variation, the dedicated study of intra-individual variation is, in view of the classical ergodic theorems, no longer an option, but a necessity."

Alternative methods are available for examining theory and data at the intra-individual level including the use of matrix algebra-based methods on multiple measures for each individual as well as Boolean algebra-based methods of the conjunctive paths occurring within individual cases (e.g., cultures, firms, decision processes, demographic-psychographic-sensemaking routes), critical discourse analysis, case-level degrees of freedom analysis, and system dynamics modeling (for discussions of all these methods and additional methods, see Woodside, 2010).

\section{THE FOUR HORSEMEN OF CASE-BASED RESEARCH}

Explicitly stating the principal objectives for case-based research serves to clarify, deepen, and impassion the investigator and her or his research. Stating four principal objectives fulfills this task. The objectives include 


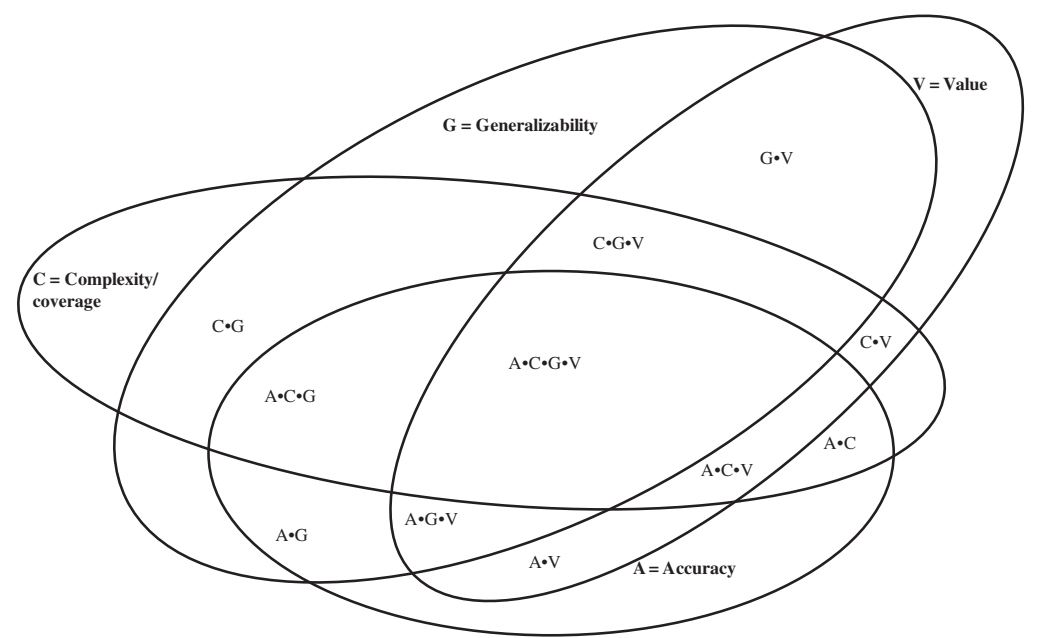

Fig. 1. The Four Horsemen of Case Study Research.

accuracy, generalizability, complexity/coverage, and value. This section briefly describes a relevant perspective for each of these objectives.

Fig. 1 is a visual of the different theoretically possible combinations of the four horsemen of case-based research. This section provides a brief platform for each.

\section{Accuracy}

Accuracy is paramount for the case study researcher (CSR). "Accuracy" in case study research is correct reporting of relevant antecedents, acts, and outcomes of a behavior (i.e., process, happening/occurrence, and/or scene); "correct" is meant to cover objective knowledge and subjective perspectives and conclusions of participants and observers of the behavior. The CSR recognizes that data collection on-site - while the respondent is enacting one or more scenes in the behavior under study - is preferable and often necessary to achieve accuracy.

The CSR recognizes that some respondents' answers to surveys include a substantial amount of subjective personal introspection (SPI) that is frequently biased, inaccurate, and incomplete in ways that result in lowering the correctness and value of the study. CSR favors transforming SPI data 
into confirmatory personal introspection (CPI) data (Woodside, 2004). "CPI" includes collecting two or more participants' interpretations and explanations while the behavior is occurring; being there - present-in-thecontext - and observing the behavior under study; and asking the participant to comment on photographs and props appearing in the context when explaining what is happening (see Mintzberg, 1979).

\section{Generalization}

Contrary to first-blush views, generalizing from a single or small number of cases is possible. Three forms of generalization apply in case-based research. Context generalization is the first form, that is, generalizing across observations within a case that includes observations (and touch points) for multiple contexts and time periods. The following vignette illustrates the idea.

Sarah is a 52 year-old adult American who lives alone. For twenty-three years and running, Sarah takes 4.5 overnight trips annually. How so? Sarah travels the 300 miles to visit her parents for six-nights per trip for four trips each year; Sarah always brings her cat, Sylvester, on these trips. Sarah takes one trip to two countries in Europe every other year; Sylvester never gets to go on these trips.

Collecting data for different situations and occurrences applicable for a given individual (e.g., person, household members, organization, firm, and nation) serves to inform how antecedent-and-process conditional statements apply in a case study. Frequently, CSR recognizes and needs to know how frequently situations occur, to learn the antecedents, processes, and outcomes for each occurrence, and to offer what-if generalizable statements that include all occurrences.

For the vignette, if a study focuses on the topic of traveling with and without pets, the following qualitative comparative analysis (QCA) written in a combination of matrix (a multiplication operation in this case) and Boolean algebra (conjunctive statements about Sarah and Sylvester) serves to compare and generalize the findings across instances within this one case:

$4($ Sarah $\bullet$ Sylvester $) \rightarrow$ Parents $)+.5($ Sarah $\bullet \sim$ Sylvester $\rightarrow$ Europe $)=$ Total annual trips.

Symbols often appearing in QCA include the mid-level dot ("•") to indicate the conjunctive "and" and the tilde symbol (" ") to indicate "not". The sideway arrow (" $\rightarrow$ ") indicates "outcome" that follows. The plus sign in Boolean algebra indicates "or." 
Generalizing from a single case to alternative theories (Campbell, 1975; Wilson \& Woodside, 1999) represent the second form of case study generalization. Famously, in 1975 Campbell revised his earlier thinking that case study research was theoretically worthless. He came to realize that the following perspective from biology and medicine also applies to theories: multiple symptoms associate with different diseases and while some of the same symptoms appear in two or more diseases, a full pattern of symptoms (e.g., 10-20 different symptoms) expresses one disease and excludes alternative diseases.

"Pattern," "path," and "recipe refers to the same proposition here: each disease and theory represents a unique set of statements that includes several hypotheses. Similar to a disease, several different indicators conjoin to form one theory versus another theory. A "critical test" (Carlsmith, Ellsworth, \& Aronson, 1976) is how well the multiple indicators within a case match with each set of overlapping statements from two or more theories. For example, Woodside and Wilson (1995) test the applicability of opposing sets of perspectives of two theories in use for representing gardening by adults with young children in the United States; Hyde and Lawson (2003) compare two opposing theories of independent travel.

Generalizing across cases is the third form of case study generalization. Research using QCA seeks to generalize across cases (see Ragin, 2008); the software program (fsQCA.com) assists in identifying the conjunctive statement - relevant pattern (causal recipe) of simple antecedent conditions relevant for each individual case and informs how frequently each path leading to an outcome under study occurs.

Consider the possibilities of different combinations of the presence or absence of six simple antecedent conditions. A total of 64 patterns (or "paths," or "recipes," or "complex antecedent combinations" of yes/no statements) are possible. Explicating (i.e., formally stating) all patterns that could occur theoretically is a step in "property space analysis" (PSA) (see Lazarsfeld, 1993); taking this step offers leads to intriguing questions and creative leaps in theory.

Empirical case-based research indicates that most cases in a set of cases (usually 10-50 cases in most case-based studies) reflect a few, not all, possible paths. Thus, with 64 possible paths, 5 paths leading to the presence of an outcome of interest might occur among 30 cases; 20 paths might occur that lead to the outcome not occurring, and no instances in the PSA occur among the remaining cases. For further details on generalizing across cases, Woodside and Zhang (2012) provide an example of using QCA to identify 
the occurrence of complex antecedent conditions leading to extremely frequent visits to casinos.

\section{Complexity/Coverage}

The objective of "complexity/coverage" refers to the depth and breadth of the occurrence of antecedents, processes, and outcomes in a case study. Achieving nuance, multiple what-if conditional statements in theory, and the reporting of infrequently occurring, yet important, rituals and rare situations follow from the achievement of high complexity/coverage.

Extended time periods (weeks, months, and sometimes years) is usually (not always) a characteristic of high complexity/coverage. Margaret Mead's (1935) Sex and Temperament in Three Primitive Societies is an early example of a participant observation case study by a trained anthropologist who lived for months within three unique Melanesian cultures: the Arapesh, the Mundungumor, and the Tchambuli.

Mead's (1935) study illustrates an attempt to achieve the complete conjunction of the four horsemen - the combination of high accuracy, generalizability, complexity/coverage, and value/impact in case study research. Stronza (2001) is a useful source reviewing the in situ stance commonly appearing in case study research seeking to achieve and report high complexity/coverage in tourism, hospitality, and leisure research.

\section{Value/Impact}

"Value/impact" is the usefulness and use of a case study report. Answers to the following issue relate to a study's value/impact. Does the study increase understanding, help solve perplexing problems, and/or help to design and implement strategies in improve government policies or marketing actions?

The relevancy of a study over decades and the number of citations appearing in the literature following a study's publication are metrics to measure a study's impact. For example, Mead's (1935) study of 3 Melanesia cultures appears in 69 editions published between 1935 and 2001 in 13 languages and the book is held by 2,082 libraries worldwide; a total of 1,746 citations to Mead (1935) appear in the literature as of November 19, 2012.

Historical analysis of a study's impact is a metric of its value. For example, possibly the most frequented visited reference (Mead, 2011, p. 1) to Mead's body of work, describes Mead's (1935) book as "a major 
cornerstone of the feminist movement [worldwide], since it claimed that females are dominant in the Tchambuli (now spelled Chambri) Lake region of the Sepik basin of Papua New Guinea (in the western Pacific) without causing any special problems."

\section{CONCLUSION}

The first two decades of the 21 st century bear witness to the coming of age of case study research. Advances in theory, methods, and practice support this conclusion and assertion.

This introductory chapter frames the content and serves to enlighten the reader as to the usefulness of case study research in general and this field guide in particular. Becoming passionate about doing case study research is more likely to follow from getting into the field - in situ contexts - quickly, with a ready guide at your side and helpful coach (see Gawande, 2011) at your back to help explain and deepen your understanding of what you are observing and doing.

Each of the authors in this field guide reports their experiences of getting into the field. We have organized the chapters here by theme, analysis of texts, executive interviews, field research, stakeholder participatory research, researching indigenous and marginal peoples, and cross-case analysis. The authors and editors intend for this book to expand your knowledge into the available methods and applications of case study research and to serve you well as your guide to undertaking such work.

\section{REFERENCES}

Campbell, D. T. (1975). Degrees of freedom in the case study research. Comparative Political Studies, 8, 178-193.

Carlsmith, J. M., Ellsworth, P. C., \& Aronson, E. (1976). Methods of research in social psychology. Reading, MA: Addison-Wesley.

Davidson, T. L. (1985). Strategic planning: A competitive necessity. The battle for market share: Strategies in research and marketing (pp. 103-108). Salt Lake City, UT: University of Utah.

Gawande, A. (2011). Personal best. The New Yorker, October 3, pp. 44-53.

Gladwell, M. (2008). Outliers: The story of success. New York, NY: Little, Brown \& Company.

Hyde, K. F., \& Lawson, R. (2003). The nature of independent travel. Journal of Travel Research, 42(1), 13-23. 
Lazarsfeld, P. F. (1993). Some remarks on typological procedures in social research. In P. F. Lazarsfeld \& R. Boudon (Eds.), Paul Z. Lazarsfeld: On social research and its language (pp. 158-167). Chicago, IL: The University of Chicago Press.

Mead, M. (1935). Sex and temperament in three primitive societies. New York, NY: Morrow.

Mead, M. (2011). Margaret Mead. http://en.wikipedia.org/wiki/Margaret_Mead

Mintzberg, H. (1979). An emergent strategy of "direct" research. Administrative Science Quarterly, 24, 582-589.

Molenaar, P. C. M., \& Campbell, C. G. (2009). The new person-specific paradigm in psychology. Current Directions in Psychological Science, 18, 112-117.

Ragin, C. C. (2008). Redesigning social inquiry: Fuzzy sets and beyond. Chicago, IL: Chicago University Press.

Stronza, A. (2001). Anthropology of tourism: Forging new ground for ecotourism and other alternatives. Annual Review of Anthropology, 30, 261-283.

Wilson, E. J., \& Woodside, A. G. (1999). Degrees-of-freedom analysis of case data in business marketing research. Industrial Marketing Management, 28, 215-229.

Woodside, A. G. (2004). Advancing from subjective to confirmatory personal introspection in consumer research. Psychology \& Marketing, 21, 987-1010.

Woodside, A. G. (2010). Case study research: Theory, methods and practice. Bingley, UK: Emerald.

Woodside, A. G. (2011). Case study research of large n survey data. In field guide to case study research in culture, tourism and hospitality research. In K. Hyde, C. Ryan \& A. G. Woodside (Eds.), Advances in culture, tourism and hospitality research (Vol. 5). Bingley, UK: Emerald.

Woodside, A. G., \& Wilson, E. J. (1995). Applying the long interview in direct marketing research. Journal of Direct Marketing, 9, 37-55.

Woodside, A. G., \& Zhang, M. (2012). Identifying X-consumers using causal recipes: "Whales" and "jumbo shrimps" casino gamblers. Journal of Gambling Studies, 28, 13-26. 